\title{
A substituição de bens de capital: um modelo de otimização sob a óptica da Engenharia de Produção
}

\author{
Replacement of capital goods: an optimzation model from the \\ perspective of Production Engineering
}

\author{
Eder Oliveira Abensur ${ }^{1}$
}

\begin{abstract}
Resumo: A substituição de bens de capital é um evento comum a todas as empresas. A decisão do momento ótimo de substituição de máquinas e equipamentos representa um evento crítico e irreversível que ocorre com grande frequência nas indústrias. Essas decisões de investimento são um dos campos tradicionais de aplicação da Engenharia Econômica. Em geral, tais decisões são tomadas por engenheiros ou por outros profissionais atuando com base nos dados levantados por engenheiros. Este trabalho desenvolveu um modelo matemático de otimização flexível e abrangente para suporte desse tipo de decisão. Paralelamente, foi feita uma análise e organização dos principais conceitos e procedimentos utilizados pelas diferentes técnicas propostas para esse tipo de decisão pelas referências adotadas por uma amostra qualitativa e relevante dos cursos de engenharia de produção brasileiros. O modelo apresentado foi denominado de RVPSE. Os testes foram feitos sobre dados reais do mercado automobilístico brasileiro, com resultados gerenciais promissores.
\end{abstract}

Palavras-chave: Substituição de equipamentos; Engenharia econômica; Programação dinâmica; Programação linear mista.

\begin{abstract}
The replacement of capital goods is an event common to all companies. The optimal replacement time of machinery and equipment represents a critical and irreversible event that occurs very frequently in industries. These investment decisions are one of the traditional fields of application of economic engineering. In general, such decisions are made by engineers or other professionals working on the basis of data provided by engineers. This study developed a flexible and comprehensive mathematical model to support this type of decision. At the same time, an analysis and organization of the main concepts and procedures were conducted, considering different techniques proposed for this type of decision by a relevant sample of Brazilian production engineering courses. The developed model was called RVPSE. The tests were performed on real data of the Brazilian automobile market with promising results.
\end{abstract}

Keywords: Equipment replacement; Economic engineering; Dynamic programming; Mixed linear programming.

\section{Introdução}

A substituição de bens de capital está entre as decisões obrigatórias e relevantes que são realizadas com frequência ao longo da vida das empresas, especialmente nas indústrias. Erros de avaliação nessas decisões podem comprometer a sobrevivência das empresas, inseridas num dinâmico ambiente competitivo.

Tanto a substituição tardia como a prematura de máquinas e equipamentos leva a organização a incorrer em perdas financeiras, respectivamente, na recuperação do capital ou nos elevados custos operacionais (Valverde \& Resende, 1997).

Praticamente todas as empresas possuem uma parte do seu capital imobilizado. A importância desses bens pode ser entendida quando consideramos que eles representam a infraestrutura produtiva da empresa, que é geradora de suas receitas.

De uma forma geral, a substituição de bens de capital deve-se a: (i) altos custos operacionais e de manutenção devido à deterioração física com idade e uso; (ii) obsolescência por avanço tecnológico que oferece métodos mais eficientes ou melhores máquinas; (iii) incapacidade de atendimento da demanda atual (inadequação); (iv) locação competitiva de equipamentos similares com vantagens fiscais; e (v) quando a substituição do equipamento, mesmo que ainda esteja em boas condições de uso, oferece alguma vantagem econômica (Blank \& Tarquin, 2008; Casarotto \& Kopittke, 2007; Ehrlich, 1986; Fleischer, 1973; Hirschfeld, 2009; Motta

\footnotetext{
${ }^{1}$ Centro de Engenharia, Modelagem e Ciências Sociais Aplicadas - CECS, Universidade Federal do ABC - UFABC, CEP 09210-170, Santo André, SP, Brasil, e-mail: eder.abensur@ufabc.edu.br
} 
\& Calôba, 2002; Newnan et al., 2002). Percebe-se a influência de fatores relacionados a desgaste por uso, obsolescência tecnológica, vantagens econômicas e fiscais.

A análise econômica da substituição de bens de capital é um projeto que envolve muitos aspectos tais como: (i) idade do bem de capital em uso; (ii) sua vida útil remanescente; (iii) custos contábeis (ex: custos de manutenção, pessoal, materiais, depreciação); (iv) custos econômicos (ex: custo de oportunidade); (v) o horizonte de planejamento para avaliação das alternativas; (vi) impostos (ex: imposto de renda); (vii) valor de aquisição do bem a ser adquirido; (viii) valor de revenda do bem em uso (se houver); $\mathrm{e}$ (ix) a taxa de desconto a ser utilizada para atualização do fluxo de caixa do projeto (Abensur, 2010).

A abordagem tradicional da substituição de bens de capital enfatiza a deterioração física do bem em uso. A ideia básica é substituir o bem quando os custos de operação e de manutenção tornam-se excessivamente altos em termos de valor presente líquido, justificando sua substituição (Suresh \& Wallace, 1992).

Entretanto, a influência da obsolescência tecnológica ganhou importância na análise de substituição como resultado de ciclos de vida de produtos cada vez menores. Essa dinâmica tecnológica comporta-se com velocidade desigual entre diferentes segmentos, sendo mais rápida, por exemplo, na indústria de microcomputadores, telefonia celular, equipamentos eletrônicos, e mais lenta em indústrias tradicionais como a automobilística. Dessa forma, vários dos modelos de substituição desenvolvidos dividem-se em conformidade com o grau de evolução tecnológica dos bens analisados. Conforme Casarotto \& Kopittke (2007), os modelos de substituição foram desenvolvidos levando-se em conta a natureza e as consequências da evolução tecnológica.

O problema da substituição de ativos ou, simplesmente, da substituição de equipamentos (PSE) é tratado tanto por escolas de administração como de engenharia. As primeiras fazem uso das técnicas de fluxo de caixa incremental (FCI), estimando os impactos de receitas, custos e impostos sobre um fluxo de caixa obtido da substituição do bem em uso (BU) pelo bem substituto (BS). O FCI é posteriormente submetido a uma avaliação por métodos de fluxo de caixa descontado (FCD), como o valor presente líquido (VPL) e/ou taxa interna de retorno (TIR) (Brigham et al., 2001; Gitman, 2010; Ross et al., 2002).

Em determinados projetos ou serviços, os benefícios ou receitas dificilmente podem ser quantificados em termos monetários, entretanto os custos podem sê-lo (Samanez, 2009). As escolas de engenharia adaptam o conceito do FCI sob a óptica dos custos envolvidos no processo de substituição. Além da obsessão da engenharia por redução de custos, esse tipo de abordagem é respaldado pelo entendimento de que os resultados serão maximizados quando os custos forem minimizados. Outra razão está associada ao comportamento mais previsível e estável dos custos em contraposição a uma conduta mais volátil e incerta das receitas. Os fluxos de custos são transformados em uma série uniforme de pagamentos pelo método do custo anual uniforme equivalente (CAUE). Os CAUEs mínimos definem a vida econômica dos bens analisados e são comparados para decidir o momento da substituição (Blank \& Tarquin, 2008; Casarotto $\&$ Kopittke, 2007; Ehrlich, 1986; Fleischer, 1973).

Os métodos baseados em FCI aplicam-se à decisão de avaliar a viabilidade econômica da substituição assumindo-se como horizonte de planejamento, em geral, a vida útil do bem em uso. Os métodos baseados em CAUE são aplicados tanto para avaliar a viabilidade econômica da troca quanto para a decisão de quando a substituição deve ser realizada, testando as possibilidades de retenção do bem em uso até a extinção de sua vida útil. Em outras palavras, os métodos de FCI procuram responder à pergunta "se", enquanto os métodos de CAUE procuram responder à pergunta "quando". Os métodos baseados em FCI fazem uso do conceito de vida útil do bem, ou seja, da sua capacidade física de produção, enquanto os métodos baseados em CAUE usam tanto a vida útil física como o conceito de vida útil econômica (VUE), que é igual ou inferior a sua vida útil. Em termos práticos, responder quando a substituição será feita já responde se ela é viável.

As primeiras publicações sobre o PSE são de meados do século XX. Esses trabalhos estabeleceram, inicialmente, a fundamentação matemática baseada em comportamentos esperados das receitas (decrescente) e dos custos envolvidos (crescente) para tratamento de dois tipos principais de situações: (i) sem inovação tecnológica; e (ii) com inovação tecnológica. A programação dinâmica (PD) foi a técnica de busca usada e considerada particularmente recomendada para esse problema. Notam-se nesses trabalhos algumas limitações de abordagem que se tornarão comuns em vários outros estudos posteriores como, por exemplo: (i) suposição de apenas uma troca durante o horizonte analisado; (ii) suposição de que o intervalo entre substituições sucessivas é constante; (iii) admissão de comportamento sempre crescente dos custos; e (iv) comparação de troca feita, em geral, contra um único bem substituto (Bellman, 1955; Dreyfus, 1960).

Ao longo dos anos, outras abordagens foram incorporadas ao PSE, as quais incluem: PD determinística, PD estocástica, cadeias de Markov, equações diferenciais etc. (Abensur, 2010; Vega \& Abensur, 2014; Derman, 1963; Ehrlich, 1986; Ekeocha et al., 2011; Hopp et al., 1987; Marques et al., 2005; Massé, 1962; Taylor, 1975; Valverde \& Resende, 1997).

Para os propósitos deste estudo, as principais fontes de informação foram extraídas das bibliografias que 
possuíam capítulos específicos sobre substituição de ativos de cursos plenos de Engenharia de Produção brasileiros, tradicionais, privados, públicos federais e estaduais reconhecidos pelos seus respectivos sistemas de avaliação de ensino e cujos programas de pós-graduação em Engenharia de Produção obtiveram, durante a avaliação trienal da Coordenação de Aperfeiçoamento de Pessoal de Nível Superior (CAPES, 2013) no período 2007-2009 conceito igual ou superior a 5 (alto desempenho) ou nota no Exame Nacional de Desempenho dos Estudantes (Enade) igual a 5 (excelente). As instituições que possuíam sua bibliografia disponível na internet e que fizeram parte da amostra foram: POLI-USP (USP, 2013a), PUC-RIO (2013), UFABC (2013), UFRGS (2013), UFSCar (2013) e USP-SÃO CARLOS (USP, 2013b). Portanto, como formadoras de opinião, elas também são consideradas como referências para a elaboração das estruturas curriculares das demais instituições de ensino brasileiras.

O propósito deste trabalho foi desenvolver um modelo matemático para suporte à clássica decisão de substituição de bens de capital. Paralelamente, foi feita uma investigação e organização conceitual da abordagem atualmente adotada por relevantes escolas de engenharia brasileiras sobre esse tipo de problema. Foram identificadas algumas controvérsias como a incorporação ou não de impostos, existência de duas técnicas distintas de análise e limitações decorrentes de simplificações feitas para facilitar os cálculos, como a comparação contra um único bem substituto.

O automóvel foi escolhido como produto de análise por representar um problema comum às pessoas físicas e jurídicas, além de possuir um mercado de negociação com valores disponíveis ao público. Os valores residuais (de mercado) dos veículos usados para teste do modelo proposto foram extraídos do caderno Classificados de Autos do jornal O Estado de S. Paulo entre 2011 e 2012. Foram selecionados veículos considerados bens substitutos em relação à potência, número de portas, acessórios, porte e preço. Por conservadorismo, os valores utilizados foram os limites inferiores dos intervalos de confiança de 95\% construídos a partir das coleções de dados levantadas. Para realização das simulações foi desenvolvido um simulador baseado em planilha Excel e no aplicativo Solver hospedado no Microsoft Excel ${ }^{\circledR}$.

A proposta de comparação sobre problemas sugeridos surgiu naturalmente como o melhor método de avaliação, pois as situações propostas replicam um significativo número de decisões distintas e factíveis de testes sobre dados reais disponíveis de mercado. Essas situações destacam a decisão de substituição de um veículo por um entre outros dois considerados similares (potência, número de portas, tamanho) fabricados por montadoras diferentes.
Quatro problemas reais de interesse para pessoas físicas e jurídicas com valores do mercado automobilístico brasileiro foram propostos para teste do modelo proposto, denominado RVPSE. Ao todo, 60 situações distintas de interesse foram avaliadas e as comparações foram feitas entre o modelo proposto e os métodos tradicionais de Engenharia Econômica.

O trabalho está estruturado da seguinte forma: (i) a seção 2 descreve os fundamentos dos métodos do CAUE; (ii) a seção 3 apresenta as principais divergências conceituais identificadas bem, como uma apreciação unificadora e fundamentada para elas; (iii) a seção 4 mostra a formulação do modelo proposto, baseado na organização conceitual realizada; (iv) na seção 5 as amostras e os testes aplicados são caracterizados bem como os resultados apresentados; e (v) a seção 6 apresenta as conclusões do estudo.

\section{Métodos baseados no custo anual uniforme equivalente}

Esta seção apresenta, de forma mais detalhada, os principais métodos de engenharia baseados no custo anual uniforme equivalente (CAUE) aplicados ao PSE. Conforme Gitman (2010), identificar os fluxos de caixa para decisões de substituição é mais complicado porque a empresa precisa identificar as saídas e entradas de caixa incremental que resultariam na troca proposta.

O PSE representa um projeto de investimento com todas as características necessárias à aplicação de técnicas de fluxo de caixa descontado (FCD) como: (i) investimento inicial para aquisição do bem substituto; (ii) entradas de caixas operacionais, depois do imposto de renda, provenientes dos bens em uso e substituto; (iii) entradas de caixa não operacionais no encerramento do projeto devido à liquidação dos bens analisados; e (iv) suposição de uma taxa mínima de atratividade (TMA), para comparação de valores monetários distribuídos em diferentes datas. Os fluxos passados, anteriores ao momento da análise da substituição, são considerados gastos irrecuperáveis e não são contemplados na análise.

Os métodos baseados no CAUE ou CAE (custo anual equivalente) ou CUL (custo uniforme líquido) são derivados do procedimento matemático de conversão do fluxo de caixa de um projeto numa série uniforme equivalente. Esse procedimento é conhecido como valor anual (VA), valor anual equivalente (VAE), anuidade equivalente (AE) ou equivalente anual (EA). Ele distribui de forma equitativa e anual a renda econômica gerada pelo projeto. Os VPLs dos projetos são transformados em séries uniformes equivalentes ao longo de sua vida útil para depois serem comparados pelos respectivos valores das AEs calculadas. Esse procedimento permite a comparação de projetos com diferentes prazos de 
duração, pois todas as AEs calculadas estão na mesma base temporal (ex: ano). Uma importante premissa conceitual implícita desse procedimento é a regra da cadeia ou do mínimo múltiplo comum, que significa a possibilidade de replicar o mesmo fluxo de caixa (ou projeto) por vários ciclos até que os horizontes de planejamento (vida útil) dos projetos analisados sejam igualados.

AAE pode ser obtida a partir da seguinte expressão (Samanez, 2009):

$$
A E=V P L\left[\frac{\left[(1+i)^{n}-1\right]}{(1+i)^{n} i}\right]
$$

em que: $i=$ custo de capital ou TMA do projeto; $n=$ prazo de duração do projeto ou vida útil.

O CAUE parte da premissa que os fluxos de caixa analisados são os custos do projeto. Outro importante conceito decorrente do CAUE é o da vida útil econômica (VUE), definida como o número, em anos, no qual ocorre a menor AE dos custos. Matematicamente, o VUE representa o ponto de mínimo da função de CAUE analisada. A obtenção da VUE é feita por tentativas sobre os valores discretos distribuídos ao longo do fluxo de caixa convencional analisado. Sob a óptica de custos, a Equação 1 pode ser reescrita conforme a Equação 2 da seguinte forma:

$$
\operatorname{CAUE}_{n, i}=\sum_{j=1}^{n}\left[\frac{C_{j}+D I_{j}}{(1+i)^{j}} \frac{\left[(1+i)^{n}-1\right]}{(1+i)^{n} i}\right]
$$

em que: $i=$ custo de capital ou TMA; $C_{j}=$ custos na data $j ; D I_{j}=$ desembolso ou investimento feito na data $j ; n=$ número de períodos analisados.

A literatura especializada apresenta vários tipos de aplicação do método CAUE ao PSE. Em alguns casos define-se um horizonte de planejamento que, em geral, coincide com a vida útil remanescente do bem em uso e sobre esse prazo determina-se qual a alternativa que apresenta o menor CAUE (Blank \& Tarquin, 2008; Hirschfeld, 2009; Samanez, 2009). Estimativas desse prazo podem ser encontradas, por exemplo, em tabelas de depreciação de bens de manuais contábeis. Em outros, o tipo de substituição é previamente classificado conforme o grau de similaridade entre os bens analisados (usa-se o termo idêntico) e a tecnologia envolvida. Esse filtro determina o tipo de análise econômica a ser efetuada que, em geral, recai sobre CAUE combinado com o conceito de VUE aplicado ao bem em uso (defensor) e ao bem substituto (desafiante) (Blank
\& Tarquin, 2008; Casarotto \& Kopittke, 2007; Ehrlich, 1986; Fleischer, 1973).

Com o intuito de facilitar a compreensão da aplicação operacional sobre os exemplos apresentados, os métodos baseados em CAUE aplicados ao PSE foram divididos em dois grupos: (i) quando nenhum horizonte de planejamento (HP) é especificado, aplica-se o conceito de vida útil econômica (VUE) associado ao CAUE; e (ii) quando um horizonte de planejamento ou vida útil funcional é especificado, aplica-se diretamente o conceito de CAUE para seleção da melhor alternativa (Blank \& Tarquin, 2008). $\mathrm{O}$ primeiro grupo foi denominado de $\mathrm{CAUE}_{(\mathrm{VUE})} \mathrm{e}$ determina o momento da troca, se houver, enquanto que o segundo grupo, denominado de $\mathrm{CAUE}_{(\mathrm{VuF})}$ define a viabilidade da substituição para o HP definido.

A Tabela 1 a seguir mostra a aplicação do método CAUE $_{(\mathrm{VUF})}$ a uma decisão de substituição de um veículo com um ano de uso, adquirido por uma pessoa física, por um modelo $0 \mathrm{~km}$ de uma outra montadora. A vida útil estimada foi de quatro anos, a TMA é de $6 \%$ a.a. e somente os dados de custos foram considerados. Sendo uma pessoa física, não há benefícios fiscais provenientes da depreciação ou dos custos operacionais. Conforme mostrado, o $\mathrm{CAUE}_{4,6 \%}$ do BU (4.269) é inferior ao $\mathrm{CAUE}_{4,6 \%}$ do BS (6.116), recomendando-se, portanto, a retenção do veículo em uso. A Tabela 2 apresenta a aplicação do

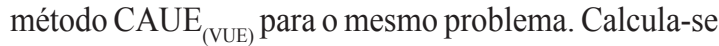
o CAUE $_{n, i}$ para cada ano de vida útil do BU e do BS. De acordo com os resultados, todos os CAUEs do BU são superiores ao do BS, recomendando-se, assim, a substituição imediata do veículo em uso.

Os métodos baseados no CAUE representam uma tentativa de recomendação sobre a viabilidade e o momento de troca do bem em uso focada exclusivamente em custos. As principais desvantagens da aplicação do $\mathrm{CAUE}_{(\mathrm{VUE})}$ e do $\mathrm{CAUE}_{(\mathrm{VUF})}$ para o PSE são: (i) o CAUE ${ }_{(\mathrm{VUE})}$ depende de que os custos envolvidos na análise definam um ponto de mínimo para aplicação do conceito de VUE; (ii) no $\mathrm{CAUE}_{(\mathrm{VUF})}$, a troca, quando recomendada, deve ser feita imediatamente, não se avaliando alguma outra possibilidade intermediária; (iii) ambos os métodos desconsideram a possibilidade de haver mais de uma troca durante o HP; (iv) por partirem de princípios distintos, o CAUE ${ }_{(\mathrm{VUE})}$ e o $\mathrm{CAUE}_{(\mathrm{VUF})}$ podem apresentar resultados divergentes para uma mesma situação (Tabelas 1 e 2); (v) implicitamente, o $\mathrm{CAUE}_{\text {(Vuf) }}$ considera que a vida útil coincide com a vida útil econômica, o que nem sempre é real; (vi) há dificuldades operacionais para tratar simultaneamente com dois ou mais bens substitutos; e (vii) o $\mathrm{CAUE}_{(\mathrm{VUE})}$ avalia a retenção do $\mathrm{BU}$ ao longo do HP e desconsidera as possíveis vantagens da relação econômica de troca com o BS. 
Tabela 1. Análise pelo método $\mathrm{CAUE}_{(\mathrm{VUF})}(\mathrm{R} \$)$.

\begin{tabular}{|c|c|c|c|c|c|c|}
\hline Manter o bem em uso (BU) & & $\mathbf{0}$ & 1 & 2 & 3 & 4 \\
\hline Valor de aquisição & 26.575 & & & & & \\
\hline TMA (\% a.a.) & $6,0 \%$ & & & & & \\
\hline IR & $0 \%$ & & & & & \\
\hline Valor residual & & & 20.242 & 19.443 & 18.473 & 17.683 \\
\hline (-) Custo de oportunidade não venda do BU & & & 1.215 & 1.167 & 1.108 & \\
\hline (-) Custo de capital & & & 6.333 & 799 & 970 & 790 \\
\hline (-) Custos operacionais & & & 4.814 & 4.422 & 4.875 & 5.239 \\
\hline \multirow[t]{3}{*}{ Incremento (a) } & & & 12.362 & 6.388 & 6.953 & 6.029 \\
\hline & & & & Venda de BU (b) & & 17.683 \\
\hline & & & & Imposto de renda (c) & & 0 \\
\hline FCI $(a-b+c)$ & & & 12.362 & 6.388 & 6.953 & -11.654 \\
\hline VP do FCI & & & 12.362 & 6.026 & 6.188 & -9.785 \\
\hline VP Total & & 14.791 & & & & \\
\hline CAUE & & 4.269 & & & & \\
\hline Trocar pelo bem substituto (BS) & & $\mathbf{0}$ & 1 & 2 & 3 & 4 \\
\hline Valor de aquisição & 26.272 & & & & & \\
\hline TMA (\% a.a.) & $6,0 \%$ & & & & & \\
\hline IR & $0 \%$ & & & & & \\
\hline Valor residual & & & 20.084 & 18.926 & 18.095 & 17.279 \\
\hline (-) Custo de oportunidade não venda do BU & & & 1.205 & 1.136 & 1.086 & \\
\hline (-) Custo de capital & & & 6.188 & 1.158 & 831 & 816 \\
\hline (-) Custos operacionais & & & 4.814 & 4.422 & 4.875 & 5.239 \\
\hline Incremento (a) & & & 12.207 & 6.716 & 6.792 & 6.055 \\
\hline (+) Compra de BS (a) & & 26.272 & & Venda de BU (b) & & 17.279 \\
\hline (-) Venda de BU (b) & & 20.242 & & Imposto de renda (c) & & 0 \\
\hline (+) IR devido a venda de BU (c) & & 0 & & & & \\
\hline$(a+b+c)$ & & 6.030 & & & $(d-e)$ & 17.279 \\
\hline FCI & & 6.030 & 12.207 & 6.716 & 6.792 & -11.224 \\
\hline VP do FCI & & 6.030 & 12.207 & 6.335 & 6.045 & -9.424 \\
\hline VP Total & & 21.193 & & & & \\
\hline CAUE & & 6.116 & & & & \\
\hline
\end{tabular}

Fonte: Adaptado de Gitman (2010) e Hirschfeld (2009).

\section{Divergências conceituais sobre a aplicação do CAUE ao PSE}

Esta seção foi motivada pela identificação de lacunas e divergências conceituais sobre o tema entre as referências usadas pelos cursos de engenharia de produção brasileiros participantes da amostra. Ela resume e apresenta as principais discrepâncias encontradas na análise conceitual efetuada.

Parte destas divergências pode ser explicada pelo tipo de abordagem ao PSE. Com o intuito de simplificar e generalizar o tema, há propostas de padronização da análise e avaliação que independem das características tecnológicas dos bens analisados (Hirschfeld, 2009), enquanto outros identificam e apresentam formas de análise de investimentos adequadas para situações predefinidas sobre o tópico de substituição de ativos físicos (Casarotto \& Kopittke, 2007; Ehrlich, 1986; Fleischer, 1973; Samanez, 2009).
A priorização do método CAUE empregado foi outro motivo observado. Alguns privilegiam a análise da viabilidade da substituição, optando pelo método CAUE $_{\text {(vuf) }}$ (Hirschfeld, 2009; Samanez, 2009). Outros priorizaram a determinação do momento da troca, adotando o método $\mathrm{CAUE}_{\text {(VUE) }}$ (Casarotto \& Kopittke, 2007; Ehrlich, 1986; Fleischer, 1973; Newnan et al., 2002). A apreciação simultânea dos dois métodos $\left(\mathrm{CAUE}_{\mathrm{(VUF}} \mathrm{e} \mathrm{CAUE}_{\mathrm{(VUE}}\right)$ foi praticada apenas por Blank \& Tarquin (2008).

Outra razão aparente está no foco dispensado pela literatura no uso de ferramental clássico de cálculo (calculadoras financeiras, planilhas eletrônicas) como apoio à aplicação dos métodos de Engenharia Econômica. Essa opção, embora coerente com a proposta de introdução a um curso de Engenharia Econômica, limita as possibilidades de busca de resultados ao PSE. A evolução e disseminação dos recursos computacionais proporcionam o tratamento do PSE por meio de modelos matemáticos alternativos 
Tabela 2. Análise pelo método $\mathrm{CAUE}_{(\mathrm{VUE})}(\mathrm{R} \$)$.

\begin{tabular}{|c|c|c|c|c|c|}
\hline Vida Econômica do BU = & 4 & & $\mathbf{E} \min =8$ & & \\
\hline Ano & & 1 & 2 & 3 & 4 \\
\hline Valor de aquisição (a) & 26.575 & & & & \\
\hline TMA (\% a.a.) & $6,0 \%$ & & & & \\
\hline IR & $0 \%$ & & & & \\
\hline Redução anual (\%) & & $16 \%$ & $7 \%$ & $5 \%$ & $3 \%$ \\
\hline Valor residual & & 20.242 & 19.443 & 18.473 & 17.683 \\
\hline Custo de capital (a) & & 6.333 & 799 & 970 & 790 \\
\hline Custos operacionais & & 4.814 & 4.422 & 4.875 & 5.239 \\
\hline VP Custos operacionais & & 4.814 & 4.172 & 4.339 & 4.399 \\
\hline VP Acumulado custos operacionais (b) & & 4.814 & 8.986 & 13.324 & 17.723 \\
\hline Custo de oportunidade (c) & & 1.215 & 1.167 & 1.108 & 1.061 \\
\hline $\mathrm{FCI}(\mathrm{d}=\mathrm{a}+\mathrm{c})$ & & 7.548 & 1.966 & 2.078 & 1.851 \\
\hline VP do FCI & & 7.548 & 1.854 & 1.850 & 1.554 \\
\hline VP FCI acumulado (e) & & 7.548 & 9.402 & 11.252 & 12.806 \\
\hline VP FCI acumulado $(b+e)$ & & 12.362 & 18.388 & 24.576 & 30.259 \\
\hline CAUE & & 13.103 & 10.029 & 9.194 & 8.810 \\
\hline Vida Econômica do BU = & 4 & & $\mathrm{E} \min =$ & & \\
\hline Ano & & 1 & 2 & 3 & 4 \\
\hline Valor de aquisição (a) & 26.272 & & & & \\
\hline TMA (\% a.a.) & $6,0 \%$ & & & & \\
\hline IR & $0 \%$ & & & & \\
\hline Redução anual (\%) & & $16 \%$ & $7 \%$ & $5 \%$ & $3 \%$ \\
\hline Valor residual & & 20.084 & 18.926 & 18.095 & 17.279 \\
\hline Custo de capital (a) & & 6.188 & 1.158 & 831 & 816 \\
\hline Custos operacionais & & 4.550 & 4.244 & 4.569 & 5.493 \\
\hline VP Custos operacionais & & 4.550 & 4.004 & 4.066 & 4.612 \\
\hline VP Acumulado custos operacionais (b) & & 4.550 & 8.554 & 12.620 & 17.232 \\
\hline Custo de oportunidade (c) & & 1.205 & 1.136 & 1.086 & 1.037 \\
\hline $\mathrm{FCI}(\mathrm{d}=\mathrm{a}+\mathrm{c})$ & & 11.943 & 6.538 & 6.486 & 7.346 \\
\hline VP do FCI & & 11.943 & 6.168 & 5.772 & 6.168 \\
\hline VP FCI acumulado (e) & & 11.943 & 18.111 & 23.883 & 30.050 \\
\hline VP FCI acumulado $(b+e)$ & & 11.943 & 18.111 & 23.883 & 30.050 \\
\hline CAUE & & 12.660 & 9.878 & 8.935 & 8.672 \\
\hline
\end{tabular}

Fonte: Elaborado pelo autor.

ou clássicos (Fleischer, 1973) que fazem uso de ferramentas computacionais sofisticadas, mas acessíveis para a busca de soluções como Microsoft Excel Solver, Solver e o LibreOffice Calc Solver (Blank \& Tarquin, 2008).

Por fim, foram verificados contrastes de enfoque sobre os custos envolvidos ao PSE oriundos de uma óptica mais contábil e fiscal contra outra mais econômica e gerencial.

\subsection{Apreciação dos custos e impostos envolvidos na análise}

Conforme visto na seção 1, todos os custos diretamente envolvidos com a decisão devem ser considerados o que, no caso do PSE, significa considerar tanto os custos variáveis (ex: operacionais) quanto os custos fixos (ex: valores de aquisição dos bens analisados). Por sua vez, os métodos de análise consideram apenas os eventos que sensibilizam o fluxo de caixa da empresa (custos marginais). Sob essa óptica, custos como a depreciação, que não representa um efetivo desembolso financeiro, devem ser excluídos da análise. Isso, no entanto, não elimina o uso da depreciação para avaliação de outros eventos como, por exemplo, os impactos fiscais de prejuízo ou lucro na venda de bens (lucro contábil).

Uma das dificuldades de análise do PSE está na apreciação simultânea de duas decisões sobre o mesmo fluxo de caixa: (i) manter o bem em uso; ou (ii) trocar por um outro bem. Essa dualidade implica na incorporação de diferentes custos, de diferentes bens em datas distintas no fluxo de caixa do projeto. Além disto, a presença dos custos de oportunidade aumenta o grau de complexidade da avaliação econômica do PSE.

As referências usadas pelos cursos de engenharia de produção brasileiros apresentam um consenso 
sobre os custos operacionais e os custos de capital. No entanto, o mesmo não ocorre com a apreciação dos custos de oportunidade e dos impactos fiscais.

A concordância sobre os custos operacionais pode ser, em parte, explicada pelo uso de dados contábeis para a análise. Há uma simplificação de todos os custos diretamente envolvidos sob contas contábeis clássicas como "operacionais" ou de "manutenção". Entretanto, sabe-se que a contabilidade tradicional apresenta dificuldades para registrar eventos produtivos intermediários, mas não enquadrados nessas classificações como, por exemplo: refugos, perdas, inadequação a novos materiais e processos, custos de set-up (preparação), custos de montagem etc. Esses custos de qualidade estão relacionados à inadequação do BU no PSE. Essa falta de segmentação de custos pode incorrer em perdas relevantes de dados e, consequentemente, em resultados subavaliados.

Em relação aos custos de oportunidade, além da inclusão dos valores de mercado do BU e BS relativos à renúncia por manter ou trocar o $\mathrm{BU}$ ao final do horizonte de planejamento, Blank \& Tarquin (2008), Fleischer (1973) e Newnan et al. (2002) também incluem os juros não recebidos pela chance perdida pela não venda do BU. Ehrlich (1986) usa os custos de inadequação como uma forma de custo de oportunidade expressa pela impossibilidade de usar um equipamento mais bem dimensionado para as novas condições de mercado. Hirschfeld (2009), Samanez (2009) e Casarotto \& Kopittke (2007) não acrescentam novos componentes ao custo de oportunidade.

Em referência à incidência do imposto de renda (IR) sobre a decisão de troca, Hirschfeld (2009) e Newnan et al. (2002) separam os efeitos em dois momentos: (i) ao fim do HP, sobre o resultado na venda do $\mathrm{BU}$, haverá receita ou despesa aparente quando ocorrer, respectivamente, prejuízo ou lucro como resultado da operação; e (ii) ao longo do HP, sobre as atividades operacionais normais também é considerada a incidência do IR sobre os custos operacionais e depreciação. Os demais autores tratam os resultados como anteriores à aplicação dos impostos.

A decisão do momento ótimo de substituição ocorre com grande frequência nas indústrias, ou seja, com pessoas jurídicas inseridas num contexto capitalista suportado por pagamento de impostos. Em muitas situações, os impostos são usados como elementos de incentivo a setores de interesse estratégico, revelando-se como fatores decisivos na análise econômica. Além disso, conforme Newnan et al. (2002), os resultados antes e após a aplicação de impostos são significativamente diferentes. Pelas razões apresentadas, a incorporação dos impostos foi considerada essencial para a análise.

As dificuldades de quantificação de alguns custos relevantes ao PSE como, por exemplo, o custo de inadequação e/ou de obsolescência, restringem a aplicação do conceito de custo de oportunidade a alguns custos identificáveis e passíveis de mensuração. Embora parcial, a incorporação desses componentes do custo de oportunidade são necessários sob um contexto de avaliação econômica de bens que oferecem o mesmo tipo de serviço, mas a custos diferentes. A Tabela 3 resume os principais eventos quantificáveis identificados e considerados pertinentes à análise do PSE.

\subsection{Classificação dos tipos de substituição}

Os projetos de substituição de bens de capital procuram analisar a viabilidade econômica da troca de um bem em uso por outros bens selecionados como potenciais substitutos. Os dados dos bens analisados são representados por características técnicas e econômicas que são incorporadas nos métodos de análise para busca de soluções. Os custos vistos na

Tabela 3. Eventos sobre o Fluxo de Caixa do PSE.

\begin{tabular}{|c|c|c|c|c|}
\hline Natureza & Tipo & Descrição & $\begin{array}{c}\text { Impacto } \\
\text { no FC }\end{array}$ & Forma de cálculo \\
\hline Custo & Operacional (CO) & MOD, materiais, manutenção etc. & $\mathrm{S}$ & Registro contábil \\
\hline Custo & Capital & $\begin{array}{l}\text { Perda de valor de mercado (VM) dos } \\
\text { bens analisados }\end{array}$ & $\mathrm{S}$ & $\mathrm{VM}_{\mathrm{i}}-\mathrm{VM}_{\mathrm{i}+1}$ \\
\hline Receita & Fiscal & $\begin{array}{l}\text { Incidência do imposto de renda } \\
\text { sobre os COs do BU e do BS }\end{array}$ & $\mathrm{E}$ & $\mathrm{IR} \times \mathrm{CO}$ \\
\hline Receita & Fiscal & $\begin{array}{l}\text { Incidência do imposto de renda } \\
\text { sobre quota de depreciação (DEP) }\end{array}$ & $\mathrm{E}$ & IR $x$ DEP \\
\hline Rec./Custo & Fiscal & $\begin{array}{l}\text { Incidência do imposto de renda } \\
\text { sobre o resultado da venda do BU } \\
\text { e/ou BS }\end{array}$ & $\mathrm{E} / \mathrm{S}$ & IR $x[$ VM - (VA - n x DEP $)]$ \\
\hline Custo & Oportunidade & Renúncia da venda do BU e/ou BS & $\mathrm{S}$ & TMA x VM \\
\hline Custo & Qualidade & Perda de qualidade, refugos etc. & $\mathrm{S}$ & Registro contábil \\
\hline
\end{tabular}

Fonte: Elaborado pelo autor. $\mathrm{E}=$ Entrada; $\mathrm{S}=$ Saída; $\mathrm{VM}_{\mathrm{i}}=$ Valor de mercado na data i; $\mathrm{VA}=$ Valor de aquisição; $\mathrm{IR}=\mathrm{Alíquota} \mathrm{do}$ imposto de renda; $\mathrm{n}=$ Idade do bem. 
seção 3.1 são exemplos de características econômicas quantificáveis. As características técnicas, no entanto, apresentam-se inseridas numa dinâmica tecnológica de inovação e diferenciação dos bens que aumenta o grau de dificuldade do PSE.

Com o intuito de organizar, definir e diferenciar os métodos de resolução aplicáveis ao PSE, vários autores identificaram situações de interesse comuns ao problema. Essas situações estão relacionadas ao tipo de similaridade técnica e econômica entre os bens, mas também podem refletir o objetivo estratégico ligado ao bem em uso.

Hirschfeld (2009), Newnan et al. (2002) e Samanez (2009) não apresentam classificações. Casarotto \& Kopittke (2007), Ehrlich (1986) e Fleischer (1973) identificaram, caracterizaram e apresentaram as formas de análise de investimentos adequadas para a avaliação econômica da viabilidade de substituição de ativos em, respectivamente, cinco, duas e quatro situações de interesse. As situações comuns são: (i) baixa sem reposição, ou seja, um ativo poderá deixar de ser econômico antes de atingir sua vida física e não ser desejável sua substituição; (ii) substituição idêntica, equipamentos que são afetados em menor escala pelo avanço tecnológico serão substituídos, devido ao desgaste, por novos equipamentos com características semelhantes; (iii) substituição não idêntica, os bens substitutos possuem alguma diferença em relação ao bem em uso, mas não há uma diferença significativa; e (iv) substituição com progresso tecnológico, os bens substitutos estimados diferem significativamente do bem em uso.

A Tabela 4 a mostra um quadro que relaciona os tipos de substituição e a decisão de retenção do BU divididos em duas etapas: (i) na etapa I compara-se a idade cronológica (IC) do BU com a vida útil estimada; e (ii) na etapa II identifica-se o método de análise recomendado e o momento ideal de troca do BU, se houver. Pelo exposto, observa-se que a classificação do PSE em tipos de substituição auxilia na organização e na identificação de características tecnológicas e econômicas dos bens participantes da análise, facilitando a seleção dos métodos de resolução adequados a cada situação.

\section{$4 \mathrm{O}$ modelo proposto}

Embora existam várias situações possíveis de serem relacionadas ao PSE (Tabela 4), elas podem ser resumidas e padronizadas, com exceção da baixa sem reposição, num modelo geral de substituição, para análise econômica, sob a óptica da representação de fluxo de caixa conforme mostrado na Figura 1. O conjunto dos tempos de vida $\left(N_{0}, N_{1}, N_{2}, \ldots, N_{K}\right)$ que minimiza o valor presente líquido do fluxo de caixa composto por saídas de custo $C_{K S}$, investimentos em novos equipamentos $I_{K}$ e valores residuais $L_{K}$ é dado pela Equação 3:

$$
V P L=\sum_{k=0}^{K}\left[\begin{array}{l}
I_{K}-L_{K}(1+i)^{-N_{K}}+ \\
\sum_{j=1}^{N_{K}} C_{K J}(1+i)^{-J}
\end{array}\right](1+i)^{-\left(\begin{array}{l}
N_{0}+N_{1}+ \\
N_{2}+\ldots .+N_{K}
\end{array}\right)}
$$

Tabela 4. Métodos de resolução relacionados ao PSE.

\begin{tabular}{|c|c|c|c|c|}
\hline Tipos de substituição & $\begin{array}{c}\text { Comparação IC } \\
\text { e VU do BU }\end{array}$ & $\begin{array}{l}\text { Decisão } \\
\text { Etapa I }\end{array}$ & $\begin{array}{c}\text { Métodos } \\
\text { recomendados }\end{array}$ & $\begin{array}{l}\text { Decisão } \\
\text { Etapa II }\end{array}$ \\
\hline \multirow[t]{2}{*}{ Baixa sem reposição } & $\mathrm{IC}>\mathrm{VU}$ & Retirada do BU & - & - \\
\hline & $\mathrm{IC}<\mathrm{VU}$ & Análise da retenção do BU & VPL & Reter até $\mathrm{VPL}<0$ \\
\hline \multirow[t]{2}{*}{ Substituição idêntica } & $\mathrm{IC}>\mathrm{VU}$ & Substituição imediata & - & - \\
\hline & $\mathrm{IC}<\mathrm{VU}$ & Análise da retenção do $\mathrm{BU}$ & $\begin{array}{l}\text { CAUE }_{(\mathrm{VUE})} \\
\text { CAUE }_{(\mathrm{VUF})}\end{array}$ & $\begin{array}{c}\text { Trocar qdo IC }=\text { VUE } \\
\text { Troca imediata } \\
\text { se CAUEBU > } \\
\text { CAUEBS }\end{array}$ \\
\hline \multirow{3}{*}{$\begin{array}{l}\text { Substituição não } \\
\text { idêntica }\end{array}$} & $\mathrm{IC}>\mathrm{VU}$ & Substituição imediata & - & - \\
\hline & $\mathrm{IC}<\mathrm{VU}$ & Análise da retenção do BU & CAUE $_{(\mathrm{VUE})}$ & $\begin{array}{c}\text { Reter enquanto } \\
\text { CAUEBU < } \\
\text { CAUEBS }\end{array}$ \\
\hline & & & $\mathrm{CAUE}_{(\mathrm{VUF})}$ & $\begin{array}{c}\text { Troca imediata } \\
\text { se CAUEBU > } \\
\text { CAUEBS }\end{array}$ \\
\hline \multirow{2}{*}{$\begin{array}{l}\text { Substituição com } \\
\text { progresso tecnológico }\end{array}$} & $\mathrm{IC}>\mathrm{VU}$ & Substituição imediata & - & - \\
\hline & $\mathrm{IC}<\mathrm{VU}$ & Análise da retenção do BU & $\begin{array}{c}\text { Programação } \\
\text { dinâmica }\end{array}$ & $\begin{array}{c}\text { Trocar quando custo } \\
\text { total da retenção } \\
>\text { custo total da } \\
\text { substituição }\end{array}$ \\
\hline
\end{tabular}


A política ótima de substituição é definida pela seleção dos valores de $N_{K}$ (intervalos entre substituições) que minimizem o VPL. Para evitar dupla contagem, as entradas devem ser líquidas de custos, ou seja, descontadas dos mesmos.

As características do fluxo de caixa da Figura 1 permitem que ele seja representado graficamente na forma de uma rede de decisões. Essa forma de representação, além de facilitar a sua compreensão, propicia o uso de eficientes técnicas de resolução como, por exemplo, os modelos de roteamento de veículos (Ahuja et al., 1993; Fisher \& Jaikumar, 1981). A cada início de estágio (ano) há duas decisões: (i) reter (R) o BU; ou (ii) substituí-lo (S). Os nós indicam a idade cronológica do BU enquanto os arcos carregam os custos de decisão em cada estágio (ver Figura 2 da seção 5).

A ferramenta de busca do modelo proposto integra a filosofia da programação dinâmica determinística em termos de análise sequencial das partes decompostas do PSE, com o algoritmo de programação linear inteira de roteirização de veículos que permite o rastreamento da melhor política de decisões inter-relacionadas. Considerando-se os custos definidos na análise da seção 3.1, as Equações 4 e 5 dos custos das decisões de reter ou substituir o bem em cada estágio (ano) podem ser descritos da seguinte maneira para uma pessoa jurídica:

a) Custo de reter o $\mathrm{BU}$

$$
\begin{aligned}
& \theta_{R}=I R C O B U_{n}^{t}+I R D e p B U_{n}^{t}- \\
& r V R B U_{n}^{t}-C C B U_{n}^{t}-C O B U_{n}^{t}
\end{aligned}
$$

b) Custo de substituir o BU

$$
\begin{aligned}
& \theta_{S}=I R C O B S i_{n}^{t}+I R D e p B S i_{n}^{t}+ \\
& \left(V R B S i_{n}^{t}-V R B U_{n}^{t}\right)-C C B S i_{n}^{t}- \\
& C O B S i_{n}^{t} \pm L U C B U_{n}^{t}
\end{aligned}
$$

em que: $B U_{n}^{t}=$ equipamento em uso com $t$ anos de idade no estágio $n ; B S i_{n}^{t}=$ equipamento substituto $i$ com $t$ anos de idade no estágio $n ; I R C O=$ imposto de renda sobre os custos operacionais; IRDep $=$ imposto de renda sobre a depreciação; $r V R=$ TMA sobre o valor residual do bem analisado; $V R B S i_{n}^{t}=$ valor residual do equipamento substituto $i \operatorname{com} t$ anos de idade no estágio $n ; V R B U_{n}^{t}=$ valor residual do equipamento em uso com $t$ anos de idade no estágio $n ; C C=$ custo de capital ou de perda de valor dos bens analisados (conforme Tabela 4); $C O=$ custos operacionais do bem analisado; $L U C=$ lucro contábil ou resultado da venda do bem.

O modelo proposto foi dividido em duas fases: (i) encontrar a política de substituições entre os bens substitutos que minimiza o custo total restrito a um determinado número de trocas $(T)$ durante um horizonte de planejamento $(H P)$; e (ii) determinar o valor de substituição (VS) caso haja retenção do BU como resultado da fase I. A função do segundo estágio é determinar o valor mínimo de mercado necessário do BU $\left(V R B U_{n}^{t}\right)$ para tornar o BS economicamente atraente a partir do custo total definido na política resultante da fase I, quando essa for de retenção do BU até o fim do HP (Blank \& Tarquin, 2008). $\mathrm{Na}$ fase II considerou-se o maior VS entre os valores encontrados dos BS analisados para troca. Os modelos matemáticos propostos são apresentados a seguir pelas Equações 6 a 13 .

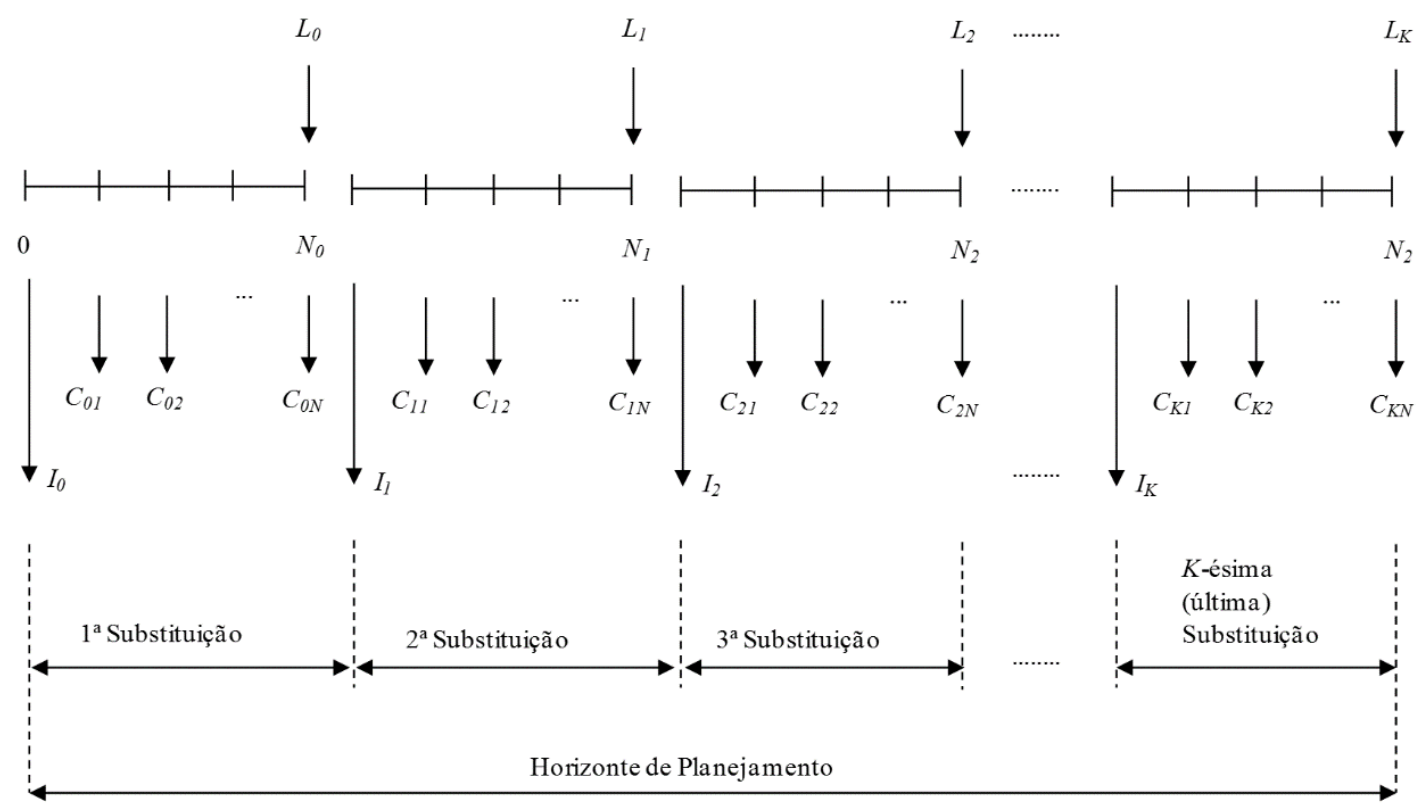

Figura 1. Modelo geral de substituição. Fonte: Adaptado de Fleischer (1973). 
Fase I:

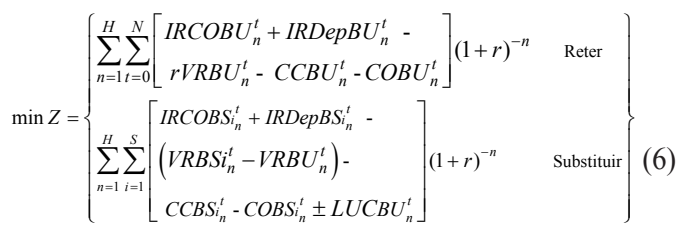

$I R C O B U_{0}^{0}=I R C O B S_{i}^{0}=I R D e p B U_{0}^{0}=$

$\operatorname{IRDepBS} S_{0}^{0}=\ldots \ldots \ldots . . .=-\operatorname{COB} U_{0}^{0}=-\operatorname{COBS} i_{0}^{0}=0$

sujeito a:

$$
\begin{aligned}
& \sum_{i=1}^{W} x_{i k}-\sum_{i=1}^{W} x_{k i}=y_{i} \\
& (\text { restrição de continuidade) } \\
& \sum_{i=1}^{H} \sum_{w=1}^{W} y_{w}=1 \\
& (\text { número de nós por estágio }) \\
& \sum_{i=1}^{H} \sum_{w=1 / w \in\{M\}}^{W} y_{w} \leq T_{i} \\
& \left(\begin{array}{l}
\text { número máximo de nós } \\
\text { de troca por estágio }
\end{array}\right) \\
& x_{i k} \in\{0,1\} \\
& y_{i} \in\{0,1\}
\end{aligned}
$$

em que: $x_{i k}=$ arco que sai de $i$ e vai para $k$ é escolhido para fazer parte da solução; $y_{j}=$ indica que o nó $i$ é escolhido para a solução; $W=$ número de nós da rede; $M=$ conjunto de nós de substituição; $S=$ número de bens substitutos em análise; $T=$ número de trocas; $N=$ maior vida útil estimada entre os bens analisados.

Fase II:

$$
\begin{aligned}
& V R B U_{n}^{t}=\theta_{R}-K \\
& \left(\begin{array}{l}
\text { Custo de trocar por BSi } \\
\text { igual ao Custo de reter o } B U
\end{array}\right) \\
& K=I R C O B S i_{n}^{t}+I R D e p B S i_{n}^{t}+ \\
& V R B S i_{n}^{t}-C C B S i_{n}^{t}-C_{B B S i}^{t} \pm L U C B U_{n}^{t}
\end{aligned}
$$

sujeito a:

$$
\begin{aligned}
& \sum_{i=1}^{W} x_{i k}-\sum_{i=1}^{W} x_{k i}=y_{i} \\
& \text { (restrição de continuidade) } \\
& \sum_{i=1}^{H} \sum_{w=1}^{W} y_{w}=1 \\
& \text { (número de nós por estágio) }
\end{aligned}
$$

$$
\begin{aligned}
& \sum_{i=1}^{H} \sum_{w=1 / w \in\{M\}}^{W} y_{w}=1 \\
& \left(\begin{array}{l}
\text { número máximo de } \\
\text { nós de troca por estágio }
\end{array}\right) \\
& V R B U_{n}^{t} \in R_{+}^{*} \\
& x_{i k} \in\{0,1\} \\
& y_{i} \in\{0,1\}
\end{aligned}
$$

A função objetivo 6 minimiza o custo total da política adotada. A Equação 7 está normalmente presente nos modelos matemáticos dos problemas de roteamento de veículos $(P R V)$ e é conhecida como de conservação do fluxo. Ela garante que a sequência dos arcos selecionados descreva uma trajetória contínua do início até o fim do horizonte de planejamento (Figura 2). As Equações 8 e 9 mostram as vantagens desse tipo de modelo, as quais incluem restrições lógicas que manipulam o número de substituições a cada estágio. Como o modelo matemático proposto representa uma adaptação de um modelo de roteamento de veículos aplicado ao problema de substituição de equipamento, ele será doravante denominado de RVPSE.

\section{Resultados}

A estrutura de rede do modelo RVPSE, baseada na lógica de decisões encadeadas da $\mathrm{PD}$, permite a avaliação da melhor política em qualquer estágio e com qualquer idade do BU. Ao todo, 60 diferentes situações de interesse foram analisadas para definição do melhor momento da substituição. Essa é uma tarefa difícil de ser replicada pelos modelos $\mathrm{CAUE}_{(\mathrm{VUE})}$ e $\mathrm{CAUE}_{(\mathrm{VUF})}$, que precisariam ser iniciados e parametrizados inúmeras vezes para o mesmo fim.

Os problemas de 1, 2 e 3 expressam a análise do momento da troca de três tipos de BU por um entre dois BS considerados para pessoas físicas e jurídicas. O problema 4 mostra o caso de uma PF que deseja trocar duas vezes seu veículo atual durante o HP por dois veículos novos do mesmo modelo e montadora. Esse último problema representa um benefício comum concedido a funcionários de montadoras instaladas no Brasil, que podem adquirir veículos novos com desconto de impostos (ICMS) durante um prazo determinado. As Tabelas 5 e 6 mostram, respectivamente, os dados essenciais de cada problema e os melhores resultados encontrados.

Algumas situações de cada problema (idade do $\mathrm{BU}=0$ no primeiro estágio) foram destacadas para avaliação de vantagens e desvantagens entre os modelos RVPSE, CAUE ${ }_{(\mathrm{VuE})}$ e CAUE $\mathrm{CVUF}_{\text {(V) }}$ Para fins de padronização de análise e comparação de resultados, os modelos CAUE $_{(\mathrm{VUE})}$ e $\mathrm{CAUE}_{\mathrm{(VUF})}$ foram aplicados conforme recomendações da literatura, com as definições apresentadas na seção 2 e com a mesma estrutura de custos mostrada na Tabela 3 da seção 3.1. A Tabela 7, a seguir, mostra as comparações feitas. Além disso, as seguintes premissas foram consideradas:

a) Não há geração direta de receita com os veículos analisados;

b) Considerou-se uma TMA de 6\% a.a. para PF e 7,5\% a.a. para $\mathrm{PJ}$

c) Adotou-se um IR de 35\% para PJ; 
d) Conforme a legislação vigente e os valores analisados, não há incidência de IR sobre os ganhos de capital para PF (Brasil, 2005);

e) Não é possível realizar mais de uma substituição no mesmo estágio (ano);

f) Os custos com combustíveis, pneus e manutenção foram considerados de acordo com os dados disponíveis das respectivas montadoras;

g) Não há o benefício da depreciação acelerada para a PJ;

h) As características do produto analisado permitem que o proprietário do veículo possa, simultaneamente, comprar um carro novo e vender o antigo pelos respectivos valores residuais aproveitando-se dos benefícios da relação de troca.

Como esperado, os resultados mostram-se coerentes e similares em quase todas as situações. Os fatores de custos envolvidos tendem a antecipar a substituição. Os custos operacionais tendem a aumentar com o tempo, o imposto de renda sobre a venda do BU atinge seu valor máximo após ele ser totalmente depreciado e o valor residual do BU é maior nos anos iniciais. A Tabela 7 mostra que quando há indicação de troca ela ocorre cedo para todos os modelos de substituição testados.

Uma flexibilidade permitida pela estrutura do RVPSE é a realização de simulações rápidas que contribuem para aumentar o conhecimento sobre o PSE. Como exemplo, o problema P1 (PJ) foi replicado sem valores residuais para os bens analisados. O resultado convergiu para a política R-S2-S1-R-R. Isso pode ser explicado, pois sem valores de mercado as substituições geram prejuízo contábil que inibem a cobrança do imposto de renda, criando, assim, um ganho econômico. Esse fato reforça a incorporação dos impactos fiscais na análise do PSE.

$\mathrm{Na}$ Tabela 7 ficam evidentes as dificuldades operacionais dos modelos $\mathrm{CAUE}_{(\mathrm{VUE})}$ e $\mathrm{CAUE}_{(\mathrm{VUF})}$ no tratamento do problema $\mathrm{P} 4$, uma vez que esses modelos não foram originalmente projetados para situações com mais de uma substituição prevista durante o HP. O modelo RVPSE avalia e manipula essa possibilidade por meio da Equação 9. Além disso, a fase II do modelo fornece o VS, uma informação gerencial útil para planejamento das possibilidades futuras de troca quando há recomendação de retenção do BU (P2).

Ao contrário dos modelos $\mathrm{CAUE}_{(\mathrm{VUE})}$ e $\mathrm{CAUE}_{(\mathrm{VUF})}$ que analisam poucas ou apenas uma combinação de substituição, o modelo RVPSE avalia todas as combinações possíveis durante o HP definido. A fase I do RVPSE é de programação linear inteira, que utiliza o algoritmo branch-and-bound, o qual possui grande confiabilidade de obtenção de solução ótima para os resultados encontrados (Taha, 2008). A fase II, de programação linear, usa o algoritmo simplex com garantia de solução ótima. Uma desvantagem aparente desse modelo está no excessivo número de possíveis combinações das políticas, que pode levar a uma dificuldade de cálculo conhecida como "maldição da dimensionalidade" (Hillier \& Lieberman, 2006;

Tabela 5. Dados dos problemas analisados.

\begin{tabular}{|c|c|c|c|c|c|c|c|c|c|c|c|}
\hline Problema & Proprietário & $\begin{array}{l}\text { Idade } \\
\text { do BU } \\
\text { (anos) }\end{array}$ & $\begin{array}{c}\mathrm{VU} \\
\text { estimada } \\
\text { (anos) }\end{array}$ & $\begin{array}{c}\text { Trocas } \\
\text { previstas }\end{array}$ & & $0^{1}$ & 1 & 2 & $\begin{array}{c}\text { Estágio } \\
3\end{array}$ & 4 & 5 \\
\hline \multirow[t]{6}{*}{$\mathrm{P} 1$} & PJ & 0 & 5 & - & $\mathrm{BU}$ & 26.272 & 22.047 & 20.083 & 18.925 & 18.095 & 17.279 \\
\hline & & & & & $\mathrm{CO}$ & - & 4.801 & 4.500 & 4.244 & 4.569 & 5.493 \\
\hline & & & & & $\mathrm{S}_{1}$ & 23.494 & 20.850 & 19.370 & 18.455 & 17.047 & 16.086 \\
\hline & & & & & $\mathrm{CO}$ & - & 3.817 & 3.699 & 3.446 & 3.784 & 4.482 \\
\hline & & & & & $\mathrm{S}_{2}$ & 26.575 & 22.105 & 20.242 & 19.443 & 18.473 & 17.683 \\
\hline & & & & & $\mathrm{CO}$ & - & 4.992 & 4.814 & 4.422 & 4.875 & 5.239 \\
\hline \multirow[t]{6}{*}{$\mathrm{P} 2$} & PJ & 0 & 5 & - & $\mathrm{BU}$ & 23.494 & 20.850 & 19.370 & 18.455 & 17.047 & 16.086 \\
\hline & & & & & $\mathrm{CO}$ & - & 3.817 & 3.699 & 3.446 & 3.784 & 4.482 \\
\hline & & & & & $\mathrm{S}_{1}$ & 26.272 & 22.047 & 20.083 & 18.925 & 18.095 & 17.279 \\
\hline & & & & & $\mathrm{CO}$ & - & 4.801 & 4.550 & 4.244 & 4.569 & 5.493 \\
\hline & & & & & $\mathrm{S}_{2}$ & 26.575 & 22.105 & 20.242 & 19.443 & 18.473 & 17.683 \\
\hline & & & & & $\mathrm{CO}$ & - & 4.992 & 4.814 & 4.422 & 4.875 & 5.239 \\
\hline \multirow[t]{6}{*}{ P3 } & $\mathrm{PF}$ & 1 & 4 & - & $\mathrm{BU}$ & 26.575 & 22.105 & 20.242 & 19.443 & 18.473 & 17.683 \\
\hline & & & & & $\mathrm{CO}$ & - & 4.992 & 4.814 & 4.422 & 4.875 & 5.239 \\
\hline & & & & & $\mathrm{S}_{1}$ & 23.494 & 20.850 & 19.370 & 18.455 & 17.047 & 16.086 \\
\hline & & & & & $\mathrm{CO}$ & - & 3.817 & 3.699 & 3.446 & 3.784 & 4.482 \\
\hline & & & & & $\mathrm{S}_{2}$ & 26.272 & 22.047 & 20.083 & 18.925 & 18.095 & 17.279 \\
\hline & & & & & $\mathrm{CO}$ & - & 4.801 & 4.500 & 4.244 & 4.569 & 5.493 \\
\hline P4 & PF & 0 & 5 & 2 & $\mathrm{BU}$ & 26.272 & 22.047 & 20.083 & 18.925 & 18.095 & 17.279 \\
\hline
\end{tabular}

${ }^{1}$ Valor de aquisição do bem. Fonte: Elaborado pelo autor. 
Tabela 6. Melhores políticas e valores de substituição (R\$).

\begin{tabular}{|c|c|c|c|c|c|c|c|}
\hline & \multirow{3}{*}{$\begin{array}{l}\text { Idade } \\
\text { do BU }\end{array}$} & \multicolumn{6}{|c|}{ Estágio } \\
\hline & & \multicolumn{2}{|c|}{1} & \multicolumn{2}{|c|}{2} & \multicolumn{2}{|c|}{3} \\
\hline & & Política & VS & Política & VS & Política & VS \\
\hline \multirow{5}{*}{ P1 } & “" $0^{1}$ & S1RRRR- & - & S1RRR & - & S1RR & - \\
\hline & 1 & S1RRRR & - & S1RRR & - & S1RR & - \\
\hline & 2 & S1RRRR & - & S1RRR & - & S1RR & - \\
\hline & 3 & RRRRR & (21.299) & S1RRR & - & S1RR & - \\
\hline & 4 & RRRRR & $(21.860)$ & S1RRR & & S1RR & - \\
\hline \multirow{5}{*}{ P2 } & 0 & RRRRR & $(25.544)$ & RRRR & $(22.675)$ & S2RR & - \\
\hline & 1 & RRRRR & $(25.524)$ & RRRR & $(22.573)$ & S2RR & - \\
\hline & 2 & RRRRR & $(24.712)$ & S2RRR & - & S2RR & - \\
\hline & 3 & RRRRR & $(22.911)$ & S1RRR & - & S2RR & - \\
\hline & 4 & RRRRR & (23.094) & S2RRR & - & S2RR & - \\
\hline \multirow{5}{*}{ P3 } & 0 & S1RRRR & - & S1RRR & - & RS1R & - \\
\hline & 1 & S1RRRR & - & S1RRR & - & RS1R & - \\
\hline & 2 & S1RRRR & - & S1RRR & - & RS1R & - \\
\hline & 3 & S1RRRR & - & S1RRR & - & RS1R & - \\
\hline & 4 & S1RRRR & - & S1RRR & - & RS1R & - \\
\hline \multirow{5}{*}{ P4 } & 0 & RSSRR & - & RSSR & - & SSR & - \\
\hline & 1 & RSSRR & - & SRSR & - & SSR & - \\
\hline & 2 & RSSRR & - & SRSR & - & SSR & - \\
\hline & 3 & RSSRR & - & SRSR & - & SSR & - \\
\hline & 4 & RSSRR & - & SRSR & - & SSR & - \\
\hline
\end{tabular}

Fonte: Elaborado pelo autor. $\mathrm{R}=$ Reter; $\mathrm{S}=$ Substituir; $\mathrm{S} 1=$ Substituir por $\mathrm{S} 1 ; \mathrm{S} 2=$ Substituir por S2; $0^{1}=$ veículo $0 \mathrm{~km}$.

Tabela 7. Comparação entre os modelos RVPSE, $\mathrm{CAUE}_{(\mathrm{VUE})}$ e $\mathrm{CAUE}_{(\mathrm{Vuf}) \text {. }}$

\begin{tabular}{|c|c|c|c|c|}
\hline & P1 & P2 & P3 & P4 \\
\hline Política RVPSE & S1RRRR & RRRRR & S1RRRR & RSSRR \\
\hline VS do BU (R\$) & - & 25.544 & - & - \\
\hline $\begin{array}{l}\text { Política CAUE }_{\text {(VuE) }} \\
\text { VUE do BU (anos) }\end{array}$ & $\begin{array}{c}\text { S1RRRR } \\
3\end{array}$ & $\begin{array}{c}\text { RRRRR } \\
2\end{array}$ & $\begin{array}{c}\text { S1RRRR } \\
4\end{array}$ & - \\
\hline Política CAUE $_{(\mathrm{VUF})}$ & S1RRRR & RRRRR & S1RRRR & - \\
\hline
\end{tabular}

Fonte: Elaborado pelo autor.

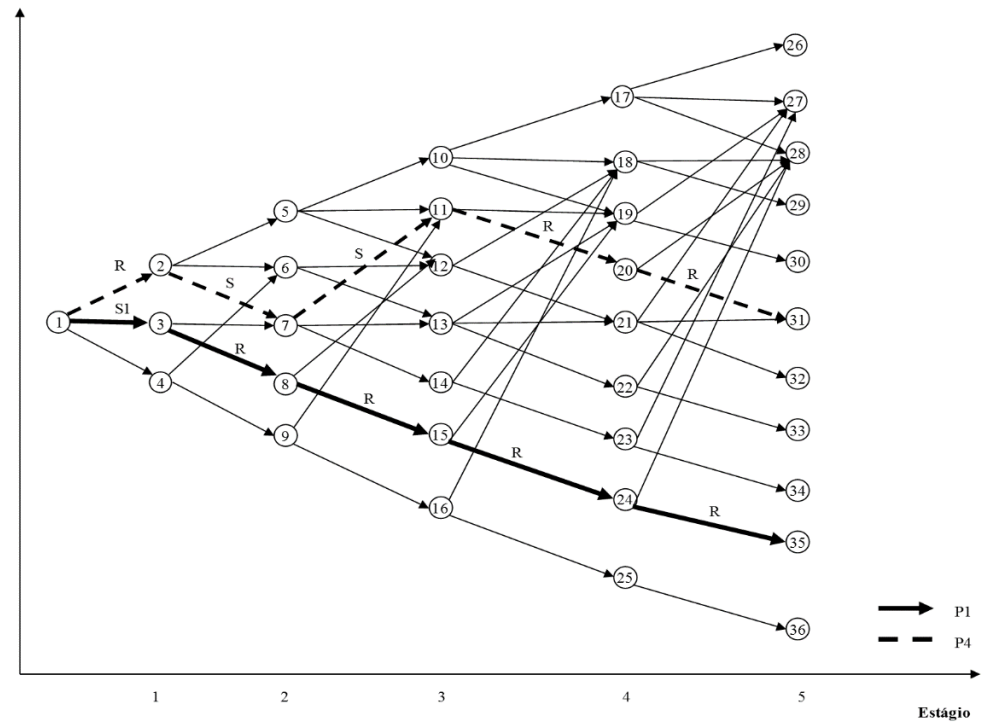

Figura 2. Rede lógica de decisão dos PSE analisados. Fonte: Elaborado pelo autor. 
Taha, 2008). No entanto, softwares globalizados livres atuais já permitem simulações com mais de mil variáveis (ex.: Calc do LibreOffice) o que permite, por exemplo, para um PSE com um BS analisado, uma autonomia significativa de 10 anos de HP.

A Figura 2 mostra as trajetórias dos problemas $\mathrm{P} 1$ e $\mathrm{P} 4$, ambos com idade do $\mathrm{BU}=0$ no primeiro estágio, na forma de rede de decisões. Além de poder lidar com mais de um BS simultaneamente e ter a flexibilidade de incorporar mais de uma substituição durante o HP, o modelo RVPSE não fica restrito a intervalos constantes entre substituições sucessivas, conforme mostrado no problema P4.

\section{Considerações finais}

Durante muito tempo o tema da substituição de bens de capital esteve acomodado dentro de uma tratativa econômica clássica com uso de ferramental operacional convencional. Da mesma forma, os conceitos e fundamentos dos métodos de análise desenvolvidos há muito não são rediscutidos sob a óptica de novos procedimentos matemáticos de otimização. Essa acomodação talvez explique a escassez de publicações, nas últimas décadas, nas principais revistas científicas brasileiras de Engenharia de Produção.

Este trabalho realizou uma análise das bibliografias usadas por reconhecidos cursos de Engenharia de Produção brasileiros sobre o tema substituição de ativos baseada nas técnicas derivadas do CAUE. Em relação a essas bibliografias verificou-se, em geral, a adoção de um único tipo de técnica de CAUE aplicada a situações convencionais restritas a uma única substituição durante o HP, comparação de troca feita contra um único bem substituto, suposição de comportamento crescente de custos, sem considerações sobre os intervalos entre substituições sucessivas e assumindo os custos antes dos impostos.

Esta pesquisa propôs um modelo matemático de otimização ( $R V P S E$ ) flexível, abrangente e aplicável a diversas situações práticas além dos problemas usualmente tratados na literatura de PSE. Esse modelo de substituição fundamentou-se nos métodos de roteamento de veículos $(P R V)$ com potenciais ganhos qualitativos e de confiabilidade em relação aos procedimentos usuais. O modelo correspondeu plenamente ao objetivo do trabalho, pois oferece aos gestores uma gama de alternativas de decisão superior aos modelos tradicionais de substituição. O modelo apresentado é inédito, considerando-se a sua sofisticação, abrangência e combinação de três tipos distintos de algoritmos adaptados ao PSE.

Vários problemas reais em Pesquisa Operacional podem ser enquadrados como problemas de roteamento (Figueredo et al., 2013). Os problemas de roteamento têm sido um dos mais abordados nas áreas de Otimização Combinatória e Pesquisa Operacional nos últimos 50 anos (Laporte, 2009). No entanto, aplicações de modelos de roteamento sobre situações distintas de transporte de produtos ainda é um campo pouco explorado na literatura. Este trabalho abre uma linha de estudo sobre adaptações de outros modelos de roteamento ao PSE e outros tipos de problema.

O produto escolhido para análise (automóvel) é de amplo uso e conhecimento de pessoas físicas e jurídicas, com um efetivo mercado de negociação. Essa escolha facilitou o levantamento de dados e a avaliação dos resultados. Uma outra linha de pesquisa aberta por esta pesquisa seria a análise da aplicação do RVPSE a outros tipos de bens, com diferente vida útil e com a respectiva avaliação dos gestores sobre os resultados.

Dada a importância do tema para a Engenharia de Produção, espera-se que este estudo sirva como um complemento bibliográfico e operacional que auxilie a abordagem praticada pelas escolas de engenharia brasileiras.

\section{Referências}

Abensur, E. O. (2010). Um modelo alternativo de otimização para a política de reposição de equipamentos. Sinergia, $11,140-150$.

Ahuja, R. K., Magnanti, T. L., \& Orlin, J. B. (1993). Network flows: theory, algorithms and applications. New Jersey: Prentice Hall.

Bellman, R. (1955). Equipment replacement policy. Journal of the Society for Industrial and Applied Mathematics, 3(3), 133-136. http://dx.doi.org/10.1137/0103011.

Blank, L., \& Tarquin, A. (2008). Engenharia econômica. São Paulo: McGraw-Hill.

Brasil. (2005, 16 de junho). Medida provisória n ${ }^{\circ}$ 252/2005, de 15 de junho de 2005. Brasília, DF: Diário Oficial da República Federativa do Brasil.

Brigham, E. F., Gapenski, L. C., \& Ehrhardt, M. C. (2001). Administração financeira: teoria e prática. São Paulo: Atlas.

Casarotto, N., Fo., \& Kopittke, B. H. (2007). Análise de Investimentos: matemática financeira, engenharia econômica, tomada de decisão, estratégia empresarial. São Paulo: Atlas.

Coordenação de Aperfeiçoamento de Pessoal de Nível Superior - CAPES. Avaliação Trienal 2010. Brasília. Recuperado em 27 de janeiro de 2013, de http://www. capes.gov.br.

Derman, C. (1963). Optimal replacement and maintenance under markovian deterioration with probability bounds and failure. Management Science, 9(3), 478-481. http:// dx.doi.org/10.1287/mnsc.9.3.478.

Dreyfus, S. E. (1960). A generalized equipment replacement study. Journal of the Society for Industrial and Applied Mathematics, 8(3), 425-435. http://dx.doi. org/10.1137/0108029. 
Ehrlich, P. J. (1986). Engenharia econômica: avaliação e seleção de projetos de investimento. São Paulo: Atlas.

Ekeocha, R. J. O., Odukwe, A. O., \& Agunwamba, J. C. (2011). Machinery replacement problems model. World Journal of Engineering and Pure and Applied Science, 1(3), 81-87.

Figueredo, G. P., Bernardino, H. S., \& Barbosa, H. J. C.. (2013). Introdução aos sistemos imunológicos artificiais. In H. S. Lopes, L. C. A. Rodrigues \& M. T. A. Steiner (Orgs.), Meta-heurísticas em pesquisa operacional (pp. 113-128). Curitiba: Omnipax.. http:// dx.doi.org/10.7436/2013.mhpo.08.

Fisher, M., \& Jaikumar, R. (1981). Generalized assignment heuristic for vehicle routing. Networks, 11(2), 109-124. http://dx.doi.org/10.1002/net.3230110205.

Fleischer, G. A. (1973). Teoria da aplicação do capital: um estudo das decisões de investimento. São Paulo: Edgard Blucher.

Gitman, L. J. (2010). Princípios de administração financeira. São Paulo: Pearson Prentice Hall.

Hillier, F. S., \& Lieberman, G. J. (2006). Introdução à pesquisa operacional. São Paulo: McGraw-Hill.

Hirschfeld, H. (2009). Engenharia econômica e análise de custos: aplicações práticas para economistas, engenheiros, analistas de investimentos e administradores. São Paulo: Atlas.

Hopp, W., Bean, J., \& Smith, R. (1987). A new optimality criteria for nonhomogeneous markov decision processes. Operations Research, 35(6), 875-883. http://dx.doi. org/10.1287/opre.35.6.875.

Laporte, G. (2009). Fifty years of vehicle routing. Transportation Science, 43(4), 408-416. http://dx.doi. org/10.1287/trsc.1090.0301.

Marques, G. M., Silva, M. L., Valverde, S. R., Noce, R., \& Castro, C. (2005). Uso de equações diferenciais para determinação do momento ótimo da transformação de veículos. Revista Árvore, 29(1), 95-104. http://dx.doi. org/10.1590/S0100-67622005000100011.

Massé, P. (1962). Optimal investment decisions: rules for action and criteria for choice. New Jersey: Prentice Hall.

Motta, R. R., \& Calôba, G. M. (2002). Análise de investimentos: tomada de decisão em projetos industriais. São Paulo: Atlas.

Newnan, D. G., Lavelle, J. P., \& Eschenbach, T. G. (2002). Essentials of engineering economic analysis. New York: Oxford University Press.

Pontifícia Universidade Católica do Rio de Janeiro - PUCRIO. Curso: Engenharia de Produção. Rio de Janeiro.
Recuperado em 27 de janeiro 2013, de http://www.pucrio.br/ensinopesq/ccg/eng_producao.html\#periodo_1.

Ross, S. A., Westerfield, R. W., \& Jaffe, J. F. (2002). Administração financeira. São Paulo: Atlas.

Samanez, C. P. (2009) Engenharia econômica. São Paulo: Pearson Prentice Hall.

Suresh, K. N., \& Wallace, J. H. (1992). A model for equipment replacement due to technological obsolescence. European Journal of Operational Research, 63(3), 207-221.

Taha, H. A. (2008). Pesquisa operacional. São Paulo: Pearson Prentice Hall.

Taylor, H. M. (1975). Optimal replacement under additive damage and other failure models. Naval Research Logistics, 22(1), 1-18. http://dx.doi.org/10.1002/ nav.3800220102.

Universidade de São Paulo - USP. Escola Politécnia da Universidade de São Paulo - POLI-USP. Departamento de Engenharia de Produção. Estrutura curricular. São Paulo. Recuperado em 27 de janeiro de 2013a, de http:// www.pro.poli.usp.br/graduacao/todas-as-disciplinas.

Universidade de São Paulo - USP. Escola de Engenharia de São Carlos. Engenharia de Produção. Disciplina: SEP0567 - Projetos de Investimento. São Paulo. Grade Curricular. Recuperado em 27 de janeiro de 2013b, de https://uspdigital.usp.br/jupiterweb/jupDisciplina?sgl dis $=$ SEP0567\& codcur $=18083 \& \operatorname{codhab}=0$.

Universidade Federal de São Carlos - UFSCar. Cursos presenciais. São Carlos. Recuperado em 27 de janeiro 2013, de http://www.prograd.ufscar.br/projetoped/ pp_engProducao_scarlos.pdf.

Universidade Federal do ABC - UFABC. Projeto pedagógico das engenharias. Santo André. Recuperado em 27 de janeiro 2013, de http://prograd.ufabc.edu.br/images/ pdf/27_01_10_projeto_pedagogico_engenharias.pdf.

Universidade Federal do Rio Grande do Sul - UFRGS. Engenharia de Produção: grade curricular. Porto Alegre. Recuperado em 27 de janeiro 2013, de http://www.ufrgs.br/ufrgs/ensino/graduacao/cursos/ exibeCurso?cod_curso $=322$.

Valverde, S. R., \& Resende, J. L. P. (1997). Substituição de máquinas e equipamentos: métodos e aplicações. Revista Árvore, 21(3), 353-364.

Vega, R. D. L., \& Abensur, E. O. (2014). Um modelo de roteamento de veículos aplicado à decisão de substituição de equipamentos: um estudo de caso do mercado automobilístico brasileiro. In Anais do $34^{\circ}$ Encontro Nacional de Engenharia de Produção. Curitiba. 